\title{
Self-Structured Organizing Single-Input CMAC Control for De-icing Robot Manipulator
}

\author{
Thanhquyen Ngo ${ }^{1,2}$, Yaonan Wang ${ }^{1}$, Youhui Chen ${ }^{1}$, Zan Xiao ${ }^{1}$ \\ ${ }^{1}$ College of Electrical and Information Engineering, Hunan University, Changsha, China \\ ${ }^{2}$ Faculty of Electrical Engineering, Ho Chi Minh City University of Industry, Ho Chi Minh, Vietnam \\ E-mail: thanhquyenngo2000@yahoo.com,yaonan@hnu.cn \\ Received June 1, 2011; revised June 22, 2011; accepted June 29, 2011
}

\begin{abstract}
This paper presents a self-structured organizing single-input control system based on differentiable cerebellar model articulation controller (CMAC) for an $n$-link robot manipulator to achieve the high-precision position tracking. In the proposed scheme, the single-input CMAC controller is solely used to control the plant, so the input space dimension of CMAC can be simplified and no conventional controller is needed. The structure of single-input CMAC will also be self-organized; that is, the layers of single-input CMAC will grow or prune systematically and their receptive functions can be automatically adjusted. The online tuning laws of single-input CMAC parameters are derived in gradient-descent learning method and the discrete-type Lyapunov function is applied to determine the learning rates of the proposed control system so that the stability of the system can be guaranteed. The simulation results of three-link De-icing robot manipulator are provided to verify the effectiveness of the proposed control methodology.
\end{abstract}

Keywords: Cerebellar Model Articulation Controller (CMAC), De-icing Robot Manipulator, Gradient-Descent Method, Self-Organizing, Signed Distance

\section{Introduction}

In general, robotic manipulators have to face various uncertainties in their dynamics, such as friction and external disturbance. It is difficult to establish exactly mathematical model for the design of a model-based control system. In order to deal with this problem, the braches of current control theories are broad including classical control: neural networks (NNs) control [1-3], adaptive fuzzy logic control (FLCs) [4-6] or adaptive fuzzy-neural networks (FNNs) [7-9] etc. They are classified as adaptive intelligent control based on conventional adaptive control techniques where fuzzy systems or neural networks are utilized to approximate a nonlinear function of the dynamical systems. However, many adaptive approaches are rejected as being overly computationally intensive because of the real-time parameter identification and required control design.

Fuzzy logic control (FLCs) has found extensive applications for plants that are complex and ill-defined which is suitable for simple second order plants. However, in case of complex higher order plants, all process states are required as fuzzy input variables to implement state feedback FLCs. All the state variables must be used to represent contents of the rule antecedent. So, it requires a huge number of control rules and much effort to create. To address these issues, single-input Fuzzy Logic controllers (S-FLC) was proposed for the identification and control of complex dynamical systems [10-12]. As a result, the number of fuzzy rules is greatly reduced compared to the case of the conventional FLCs, but its control performance is almost the same as conventional FLCs.

Neural networks (NNs) are a model-free approach, which can approximate a nonlinear function to arbitrary accuracy [1-3]. However, the learning speed of the NNs is slow. To deal with these issues, cerebellar model articulation controller (CMAC) was proposed by Albus in 1975 [13] for the identification and control of complex dynamical systems, due to its advantage of fast learning property, good generalization capability and ease of implementation by hardware [13-15]. The conventional CMACs, regarded as non-fully connected perceptron-like associative memory network with overlapping receptive fields which used constant binary or triangular functions. The disadvantage is that their derivative information is not preserved. For acquiring the derivative information of 
input and output variables, Chiang and Lin [16] developed a CMAC network with a differentiable Gaussian receptive-field basis function and provided the convergence analysis for this network. The advantages of using CMAC over neural network in many applications were well documented [17-21]. However, in the above CMAC literatures, the structure of CMAC cannot be obtained automatically. The amount of memory space is difficult to select, which will influence the learning and control schemes. Some self-organizing CMAC neural networks were proposed for structure adaptation [22-25]. In [22] and [23] a data clustering technique is used to reduce the memory size and a structural adaptation technique is developed in order to accommodate new data sets. However, only the structure growing mechanism is introduced and; the pruning mechanism was not discussed. In [24], a self-organizing hierarchical CMAC was introduced. The authors proposed a multilayer hierarchical CMAC model and used Shannon's entropy measure and golden-section search method to determine the input space quantization. However, their approach is too complicated and lacks online real-time adaptation ability. Online adjusting suitable memory space of CMAC structure is our motivation. To address these issues, C. M. Lin, T. Y. Chen proposed self-organizing control system [25]. This control system does not require prior knowledge amount of memory space, the layers of CMAC will grow or prune systematically. However, the dimension of the input space of CMAC control system is reduced through a combination of sliding control model. Recently, to deal with the problem of the simplified input, B. J Choi, S. W. Kwak and B. K. Kim proposed the S-FLC [10-12] and its advantages which are mentioned above. Based on the S-FLC, several literatures developed single-input CMAC (S-CMAC) control system [26,27], which adopts two learning stages, namely, an offline learning stage and online learning stage. The disadvantage is that their derivative information is also not preserved. So, M. F. Yeh and C. H. Tsai proposed differentiable standalone CMAC control system [28] to provided better system status in the learning control. In addition, the quantization of input space could be reduced while using the differentiable standalone CMAC. However, the disadvantages are that the structure of S-CMAC cannot be obtained automatically.

In this paper, we propose a novel self-structured organizing single-input CMAC (SOSICM) control system for three-link De-icing robot manipulator to achieve the high-precision position tracking. This control system combines advantages of S-CMAC and it does not require prior knowledge of a certain amount of memory space, and the self-organizing approach demonstrates the properties of generating and pruning the input layers auto- matically. The developed self-organizing rule of S-CMAC is clearly and easily used for real-time systems. Moreover, the developed system is solely used to control the plant and no conventional or compensated controller. The online tuning laws of CMAC parameters are derived in gradient-descent method.

This paper is organized as follows: System description is described in section 2. Section 3 presents SOSICM control system. Numerical simulation results of a three-link De-icing robot manipulator under the possible occurrence of uncertainties are provided to demonstrate the tracking control performance of the proposed SOSICM system in section 4. Finally, conclusions are drawn in section 5 .

\section{System Description}

In general, the dynamic of an $n$-link robot manipulator may be expressed in the Lagrange following form:

$$
M(q) \ddot{q}+C(q, \dot{q}) \dot{q}+G(q)+N=\tau
$$

where $q, \dot{q}, \ddot{q} \in R^{n}$ are the joint position, velocity and acceleration vectors, respectively, $M(q) \in R^{n \times n}$ denotes the inertia matrix, $C(q, \dot{q}) \in R^{n \times n}$ expresses the matrix of centripetal and Coriolis forces, $G(q) \in R^{n \times 1}$ is the gravity vector, $N \in R^{n \times 1}$ represents the vector of external disturbance $t_{l}$, friction term $f(\dot{q})$, and un-modeled dynamics, $\tau \in R^{m \times 1}$ is the torque vectors exerting on joints. In this paper, a new three-link De-icing robot manipulator, as shown in Figure 1, is utilized to verify dynamic properties are given in section 4 .

The control problem is to force $q_{i}(t) \in R^{n}$, $i=1,2, \cdots m$ to track a given bounded reference input signal $q_{d i}(t) \in R^{n}$. Let $e_{i}(t)$ be the tracking error vector as follows:

$$
e_{i}=q_{d i}-q_{i}, \quad i=1,2, \cdots m
$$

and the system tracking error vector is defined as

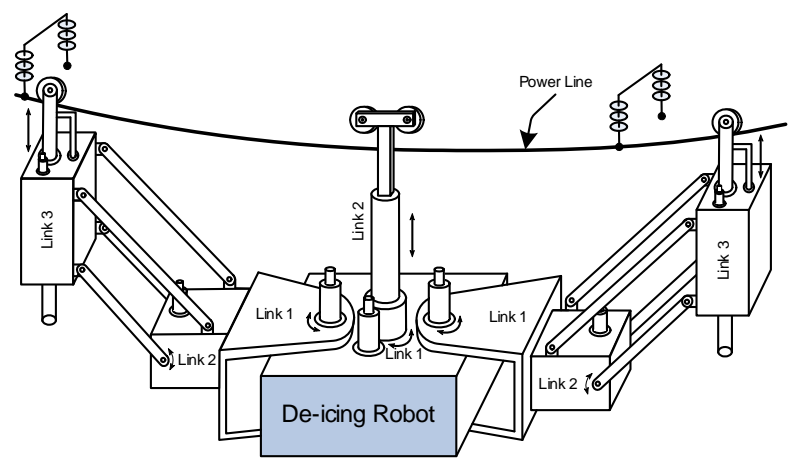

Figure 1. Architecture of three-link De-icing robot manipulator. 


$$
\begin{aligned}
\varepsilon_{i} & =\left[\begin{array}{cccc}
k_{1 i} & 0 & \cdots & 0 \\
0 & k_{2 i} & \cdots & 0 \\
0 & 0 & \cdots & 0 \\
0 & 0 & \cdots & k_{n i}
\end{array}\right]\left[\begin{array}{c}
e_{i} \\
\dot{e}_{i} \\
\vdots \\
e_{i}^{n-1}
\end{array}\right] \\
& =\left[\begin{array}{llll}
k_{1 i} e_{i} & k_{2 i} \dot{e}_{i} & \cdots & k_{n i} e_{i}^{n-1}
\end{array}\right] \\
& =\left[\begin{array}{llll}
\varepsilon_{1 i} & \dot{\varepsilon}_{2 i} & \cdots & \varepsilon_{n i}^{n-1}
\end{array}\right], \quad i=1,2, \cdots, m
\end{aligned}
$$

where $K_{n i} \in R^{n \times n}$ is the scaling factor matrix for the

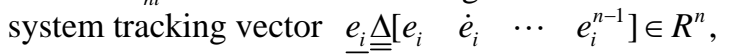
$i=1,2, \cdots m$.

Based on $[10,11]$, the tracking error $\varepsilon_{i} \in R^{n}$ is transformed into a single variable, termed the signed distance $d_{s i} \in R^{m}$, which is the distance from an actual state $\varepsilon_{i} \in R^{n}$ to the switching line as shown in Figure 2 for a 2-D input. The switching line is defined as follows:

$$
e_{i}^{n-1}+\lambda_{n-1} e_{i}^{n-2}+\cdots+\lambda_{2} \dot{e}_{i}+\lambda_{1} e_{i}=0
$$

where $\lambda_{n-1} \in R^{n-1}$ is a constant. Then, the signed distance between the switching line and operating point $\varepsilon_{i} \in R^{n}$ can be expressed by the following equation:

$$
d_{s i}=\frac{\varepsilon_{n i}^{n-1}+\lambda_{n-1} \varepsilon_{(n-1) i}^{n-2}+\cdots+\lambda_{2} \dot{\varepsilon}_{2 i}+\lambda_{1} \varepsilon_{1 i}}{\sqrt{1+\lambda_{n-1}^{2}+\cdots+\lambda_{2}^{2}+\lambda_{1}^{2}}}
$$

According to the standalone CMAC control system is shown in Figure 3. This control scheme provided better

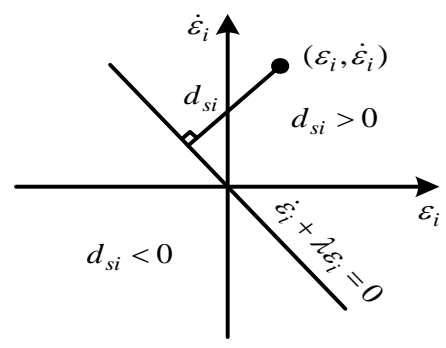

Figure 2. Derivation of a signed distance.

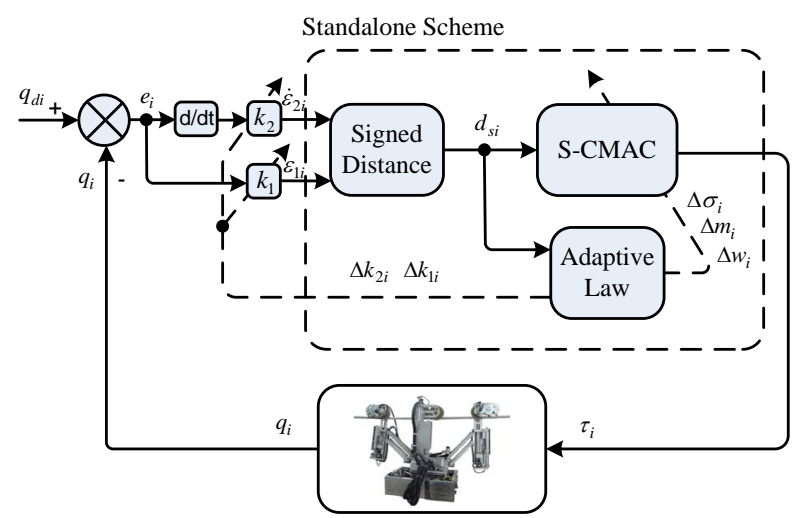

Figure 3. Block diagram of standalone CMAC control system. control characteristics due to using the differentiable CMAC in the system. The advantage is that derivative information of input and output variables is preserved in learning process. In addition, the generalization error caused by quantization of input space could be reduced while using the differentiable CMAC.

Based on the standalone CMAC control system, we propose the SOSICM control system as shown in Figure 4, which combines advantages of standalone CMAC and it does not require prior knowledge of a certain amount of memory space. The self-organizing approach demonstrates the properties of generating and pruning the input layers automatically. The developed self-organizing rule of CMAC is clearly and easily used for real-time systems

\section{Adaptive SOSICM Control System}

\subsection{Brief of the S-CMAC}

An S-CMAC is proposed and shown in Figure 5. It is composed of an input, association memory, weight and output spaces. The signal propagation and the basic function in each space are expressed as follows:

1) Input space $D_{s}$; assume that each input state variable $d_{s i}$ can be quantized into $N_{s i}$ discrete states and that the information of a quantized state is regarded as region for each layer $n_{k i}$ th . Therefore, there exist $N_{s i}+1$ individual points on the $d_{s i}$ - axis. Figure 6 shows the case of $N_{s i}=10$. Each activated state in each layer becomes a firing element, thus, the weight of each layer can be obtained. The Gaussian basic function for each layer is given as follows:

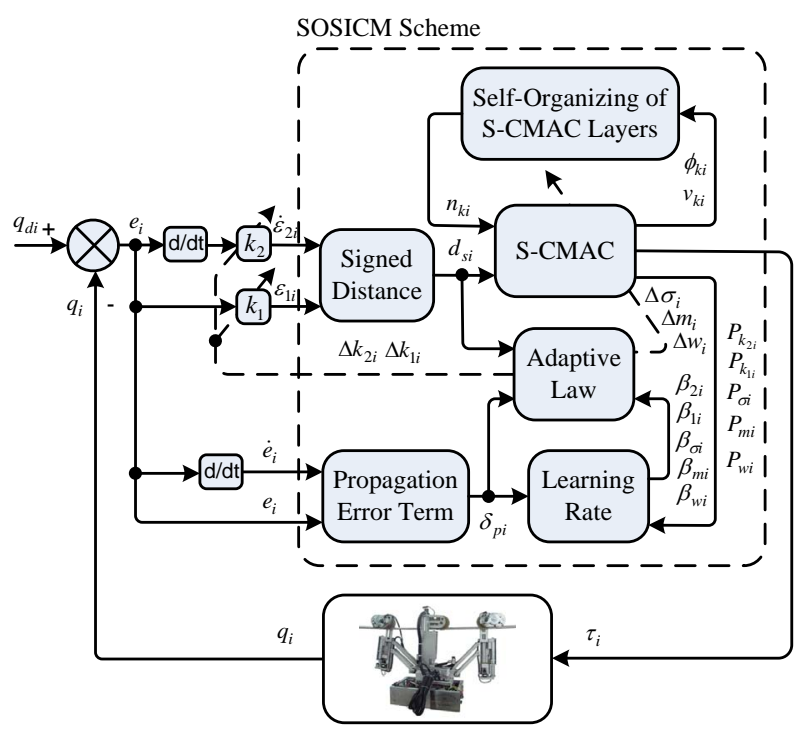

Figure 4. Block diagram of proposed SOSICM control system. 


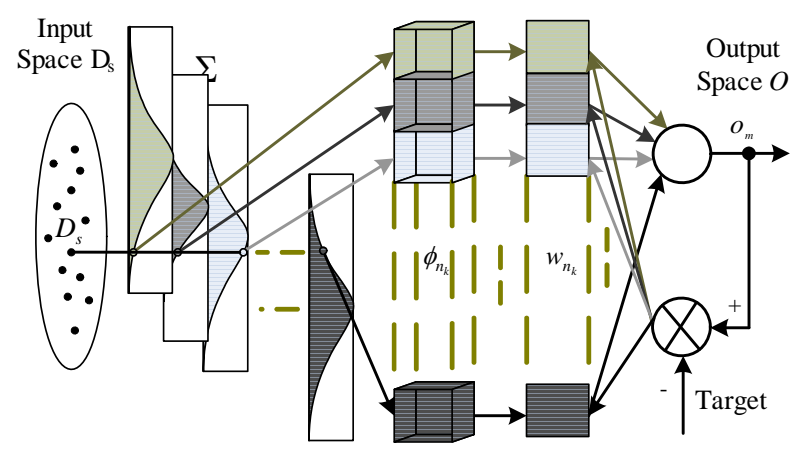

Association Memory Space $A$ Weight Memory Space $W$

Figure 5. Architecture of a single-input CMAC.

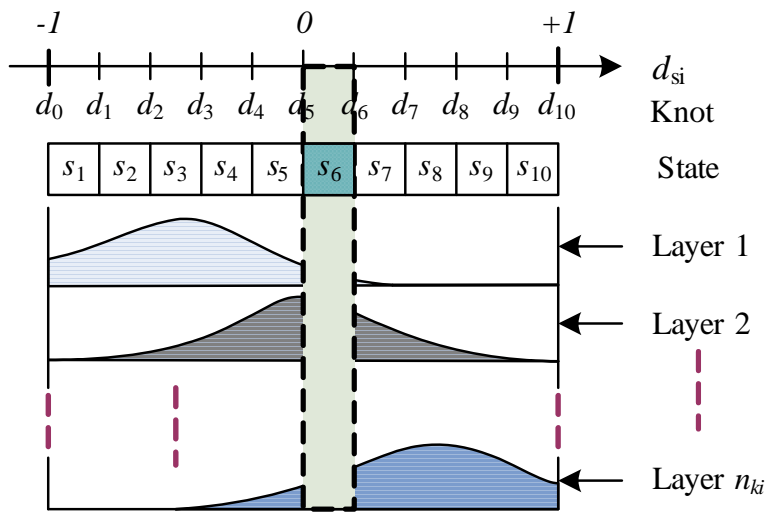

Figure 6. Block division of CMAC with Gaussian basic function.

$$
\begin{aligned}
& \varphi_{k i}\left(d_{s i}\right)=\exp \left[\frac{\left(d_{s i}-m_{k i}\right)^{2}}{\sigma_{k i}^{2}}\right], \\
& i=1,2, \cdots, m, \quad k=1,2, \cdots, n_{k i}
\end{aligned}
$$

where $\phi_{k i}$ represents the $k$ th layer of the input $d_{s i}$ with the mean $m_{k i}$ and the variance $\sigma_{k i}$.

2) Output space O: The output of S-CMAC is the algebraic sum of the firing element with the weight memory, and is expressed as

$$
\tau_{i}=\sum_{k=1}^{n_{k i}} a_{k i} w_{k i} \phi_{k i}\left(d_{s i}\right)
$$

where $w_{k i}$ denotes the weight of the $k$ th layer, $a_{k i}=a_{k i}\left(d_{s i}\right), \quad k=1,2, \cdots n_{k i}$ is the index indicating whether the ith memory element is addressed by the state involving $d_{s i}$. Since each state addressed exactly $m_{k i}$ memory elements, only those addressed $a_{k i}$ s are one, and the others are zero.

The block diagram in Figure 3, in which only the $\mathrm{S}$-CMAC plays a major role in the control process, thus to have a trade-off between the desired performance and the computation loading we must choose a reasonable number of layers. However, if the number of layers is chosen too small, the learning performance may be insufficient to achieve a desired performance. Otherwise, if the number of layers is chosen too large, the calculation process is too heavy, so it is not suitable for real-time applications. To deal with this problem, a self-structured organizing S-CMAC is proposed includes structure and parameter learning as shown in Figure 4.

\subsection{Self-Structured Organizing S-CMAC}

In this section, structural learning is necessary to determine whether to add a new layer in association memory $A$ depends on the firing strength $\phi_{k i} \in R^{n_{k i}}$ of each layer for each incoming data $d_{s i}$. If the firing strength $\phi_{k i} \in R^{n_{k i}}$ of each layer for new input data $d_{s i}$ falls outside the bounds of the threshold, then, SOSICM will generate a new layer. The self-structured organizing S-CMAC can be summarized as follows:

1) Calculate the firing strength $\phi_{k i} \in R^{n_{k i}}$ of each layer for each input data $d_{s i}$ in (6).

2) Using Max-Min method is proposed for layer growing. Find

$$
\hat{k}_{i}=\arg \min _{1 \leq k \leq n_{k i}} \phi_{k i}\left(d_{s i}\right), k=1,2, \cdots n_{k i}
$$

If

$$
\phi_{\hat{k}_{i}}\left(d_{s i}\right) \phi<K_{g i}
$$

Here $K_{g i}$ is a threshold value of adaptation with $0<K_{g i} \leq 1$. In our case $K_{g i}=0.1$ and a new layer is generated.

This means that for a new input data, the exciting value of existing basic function is too small. In this case, number of layers increased as follows:

$$
n_{k i}(t+1)=n_{k i}(t)+1
$$

where $n_{k i}$ is the number of layers at time $t$. Thus, a new layer will be generated and then the corresponding parameters in the new layer such as the initial mean and variance of Gaussian basic function in association memory space and the weight memory space will be defined as

$$
\begin{aligned}
& m_{n_{k i}}=d_{s i} \\
& \sigma_{n_{k i}}=\sigma_{\hat{k} i} \\
& w_{n_{k i}}=0
\end{aligned}
$$

Another self-structured organizing learning process is considered to determine whether to delete existing layer, which is inappropriate. A Max-Min method is proposed for layer pruning.

Considering the output of SOSICM in (7), the ratio of the $k$ th component of output is defined as 


$$
M M_{k i}=\frac{v_{k i}}{\tau_{i}}, k=1,2, \cdots, n_{k i}
$$

where $v_{k i}=\phi_{k i} w_{k i}$, Then, the minimum ratio of the $k$ th component is defined as follows:

$$
\tilde{k}_{i}=\arg \min _{1 \leq k \leq n_{k i}} M M_{k i}
$$

If

$$
M M_{\tilde{k_{i}}} \leq K_{c i}
$$

Here $K_{c i}$ is a predefined deleting threshold. In our case $K_{c i}=0.03$ and the $\tilde{k}_{i}$ th layer will be deleted. This means that for an output data, if the minimum contribution of a layer is less than the deleting threshold, then this layer will be deleted.

\subsection{On-Line Learning Algorithm}

The central part of the learning algorithm for a SOSICM is how to choose the weight memory $w_{k i}$, mean $m_{k i}$, variance $\sigma_{k i}$ of the Gaussian basic function. $k_{n i}$ Are the scaling factors of the error $e_{i}$ and the change of error $\dot{e}_{i}$, which will significantly affect the performance of SOSICM. For achieving effective learning, an on-line learning algorithm, which is derived using the supervised gradient descent method, is introduced so that it can in real-time adjust the parameters of SOSICM. The energy function $E_{i}$ is defined as

$$
E_{i}=\frac{1}{2}\left(q_{d i}-q_{i}\right)^{2}=\frac{1}{2} e_{i}^{2}
$$

According to the energy function (17) and the system structure in Figure 4, the error term to be propagated is given by

$$
\delta_{p i}=-\frac{\partial E_{i}}{\partial \tau_{i}}=-\frac{\partial E_{i}}{\partial q_{i}} \frac{\partial q_{i}}{\partial \tau_{i}}=e_{i} \frac{\partial q_{i}}{\partial \tau_{i}}
$$

where $\partial q_{i} / \partial \tau_{i}$ represent the sensitivity of the plant with respect to its input. With the energy function $E_{i}$, the parameters updating law based on the normalized gradient descent method can be derived as follows

1) The updating law for the kth weight memory can be derived according to

$$
\begin{aligned}
\Delta w_{k i} & =-\beta_{w i} \frac{\partial E_{i}}{\partial w_{k i}}=-\beta_{w i} \frac{\partial E_{i}}{\partial \tau_{i}} \frac{\partial \tau_{i}}{\partial w_{k i}} \\
& =a_{k i} \beta_{w i} \delta_{p i} \varphi_{k i}\left(d_{s i}\right)
\end{aligned}
$$

where $\beta_{w i}$ is positive learning rate for the output weight memory $w_{k i}$, the connective weight can be updated according to the following equation:

$$
w_{k i}(t+1)=w_{k i}(t)+\Delta w_{k i}
$$

2) The mean and variance of the kth Gaussian basic function can be also updated according to

$$
\begin{aligned}
\Delta m_{k i} & =-\beta_{m i} \frac{\partial E_{i}}{\partial m_{k i}}=-\beta_{w i} \frac{\partial E_{i}}{\partial \tau_{i}} \frac{\partial \tau_{i}}{\partial m_{k i}} \\
& =a_{k i} \beta_{m i} \delta_{p i} w_{k i} \varphi_{k i}\left(d_{s i}\right) \frac{2\left(d_{s i}-m_{k i}\right)^{2}}{\sigma_{k i}^{2}} \\
\Delta \sigma_{k i} & =-\beta_{\sigma i} \frac{\partial E_{i}}{\partial \sigma_{k i}}=-\beta_{w i} \frac{\partial E_{i}}{\partial \tau_{i}} \frac{\partial \tau_{i}}{\partial \sigma_{k i}} \\
& =a_{k i} \beta_{\sigma i} \delta_{p i} w_{k i} \varphi_{k i}\left(d_{s i}\right) \frac{2\left(d_{s i}-m_{k i}\right)^{2}}{\sigma_{k i}^{3}}
\end{aligned}
$$

where $\beta_{m i}, \beta_{\sigma i}$ are positive learning rates for the mean and variance, respectively. The mean and variance can be updated as follows:

$$
\begin{gathered}
m_{k i}(t+1)=m_{k i}(t)+\Delta m_{k i} \\
\sigma_{k i}(t+1)=\sigma_{k i}(t)+\Delta \sigma_{k i}
\end{gathered}
$$

3) Finally, the updating law for scaling factors can be derived as follows:

$$
\begin{aligned}
\Delta k_{n i}= & -\beta_{n i} \frac{\partial E_{i}}{\partial k_{n i}}=-\beta_{n i} \frac{\partial E_{i}}{\partial \tau_{i}} \frac{\partial \tau_{i}}{\partial d_{s i}} \frac{d_{s i}}{k_{n i}} \\
= & \beta_{n i} \delta_{p i}\left[\sum_{k=1}^{n_{k i}} a_{k i} w_{k i} \phi_{k i}\left(d_{s i}\right) \frac{-2\left(d_{s i}-m_{k i}\right)}{\sigma_{k i}^{2}}\right] \\
& \frac{\lambda_{n} e_{n i}^{n-1}}{\sqrt{1+\lambda_{n-1}^{2}+\cdots+\lambda_{2}^{2}+\lambda_{1}^{2}}}
\end{aligned}
$$

where $\beta_{m i}$ is the learning rate, and it can be updated by the following:

$$
k_{n i}(t+1)=k_{n i}(t)+\Delta k_{n i}
$$

The plant sensitivity $\partial q_{i} / \partial \tau_{i}$ in (18) can be calculated if the plant model is exactly known. However, the plant model is unknown, so $\partial q_{i} / \partial \tau_{i}$ can not obtained in advance. To deal with this problem, in [28], a simple approximation of the error term of the system can be use as follows:

$$
\delta_{p i} \cong \dot{e}_{i}+e_{i}
$$

\subsection{Convergence Analysis}

The update laws of Equations (19), (21), (22) and (25) require a proper choice of the learning rates $\beta_{w i}, \beta_{m i}$, $\beta_{\sigma i}$, and $\beta_{n i}$ in order to the convergence of the output error is guaranteed; however, this is not easy which depends on each person's experience. To train the S-CMAC effectively, the variable learning rates which guarantee convergence of the output error are derived in the following.

Defined a discrete-type Lyapunov function can be 
given by

$$
V_{i}(k)=\frac{1}{2} e_{i}^{2}(k)
$$

Thus, the change of the Lyapunov due to the training process is obtained as

$$
\Delta V_{i}(k)=V_{i}(k+1)-V_{i}(k)=\frac{1}{2}\left[e_{i}^{2}(k+1)-e_{i}^{2}(k)\right]
$$

where $e_{i}(k+1)$ is represented by [28]

$$
e_{i}(k+1)=e_{i}(k)+\Delta e_{i}(k)=e_{i}(k)+\left[\frac{\partial e_{i}(k)}{\partial P_{i}}\right]^{T} \Delta P_{i}
$$

where $\Delta e_{i}$ represents the in the learning process, $\Delta P_{i}$ denotes a change of an adjustable parameters. Using Equation (18), we have $\partial e_{i} / \partial P_{i}=-\delta_{p i} \partial \tau_{i} / e_{i}(k) \partial P_{i}$ and $\Delta P_{i}=-\beta_{p i} \partial E_{i} / \partial P_{i}=\beta_{p i} \delta_{p i} \partial \tau_{i} / \partial P_{i}$, where $\beta_{p i}$ is the learning rate for the parameter $P_{i}$.

Thus:

$$
\begin{aligned}
\Delta V_{i}(k) & =\Delta e_{i}(k)\left[e_{i}(k)+\frac{1}{2} \Delta e_{i}(k)\right] \\
& =-\frac{\beta_{p i} \delta_{p i}^{2}}{e_{i}(k)}\left\|\frac{\partial \tau_{i}}{\partial P_{i}}\right\|^{2}\left[e_{i}(k)-\frac{1}{2} \frac{\beta_{p i} \delta_{p i}^{2}}{e_{i}(k)}\left\|\frac{\partial \tau_{i}}{\partial P_{i}}\right\|^{2}\right] \\
& =\frac{1}{2} \beta_{p i} \delta_{p i}^{2}\left\|\frac{\partial \tau_{i}}{\partial P_{i}}\right\|^{2}\left[\beta_{p i}\left(\frac{\delta_{p i}}{e_{i}(k)}\right)^{2}\left\|\frac{\partial \tau_{i}}{\partial P_{i}}\right\|^{2}-2\right]
\end{aligned}
$$

If the learning rate $\beta_{p i}$ is selected as:

$$
0<\beta_{p i}<2 /\left[\delta_{p i} / e_{i}(k)\right]^{2}\left\|\partial \tau_{i} / \partial P_{i}\right\|^{2}
$$

Then $\Delta V_{i}(k) \leq 0$, therefore $V_{i}(k+1) \leq V_{i}(k)$, the Lyapunov stability (system stability) and the convergence of the tracking error could be guaranteed. In addition, the optimal learning rate can be found for achieving faster convergence by taking the differential equation (31) with respect to $\beta_{p i}$ and equals to zero. Finally, the optimal learning rate can be determined as follows:

$$
\beta_{p i}^{*}=1 /\left[\delta_{p i} / e_{i}(k)\right]^{2}\left\|\partial \tau_{i} / \partial P_{i}\right\|^{2}
$$

where $\partial \tau_{i} / \partial P_{i}$ for $P_{i}=w_{k i}, m_{k i}, \sigma_{k i}$ and $k_{n i}$, it can be obtained as:

$$
\begin{gathered}
P_{w i}(k)=\frac{\partial \tau_{i}}{\partial w_{k i}}=a_{k i} \phi_{k i}, \\
P_{m i}(k)=\frac{\partial \tau_{i}}{\partial m_{k i}}=a_{k i} w_{k i} \phi_{k i} \frac{2\left(d_{s i}-m_{k i}\right)}{\sigma_{k i}^{2}} \\
P_{\sigma i}(k)=\frac{\partial \tau_{i}}{\partial \sigma_{k i}}=a_{k i} w_{k i} \varphi_{k i} \frac{2\left(d_{s i}-m_{k i}\right)^{2}}{\sigma_{k i}^{3}}
\end{gathered}
$$

$$
\begin{gathered}
P_{k_{n i}}(k)=\frac{\partial \tau_{i}}{\partial k_{n i}}=\left[\sum_{k=1}^{n_{k i}} a_{k i} w_{k i} \phi_{k i}\left(d_{s i}\right) \frac{-2\left(d_{s i}-m_{k i}\right)}{\sigma_{k i}^{2}}\right] \\
\\
\frac{\lambda_{n} e_{n i}^{n-1}}{\sqrt{1+\lambda_{n-1}^{2}+\cdots+\lambda_{2}^{2}+\lambda_{1}^{2}}}
\end{gathered}
$$

\section{Simulation Results}

A three-link De-icing robot manipulator as shown in Figure 1 is utilized in this paper to verify the effectiveness of the proposed control scheme. The detailed system parameters of this robot manipulator are given as: link mass $m_{1}, m_{2}, m_{3}(\mathrm{~kg})$, lengths $l_{1}, l_{2}(m)$, angular positions $q_{1}, q_{2}(\mathrm{rad})$ and displacement position $d_{3}(m)$.

The parameters for the equation of motion (1) can be represented as follow:

$$
\begin{gathered}
M(q)=\left[\begin{array}{lll}
M_{11} & M_{12} & M_{13} \\
M_{21} & M_{22} & M_{23} \\
M_{31} & M_{32} & M_{33}
\end{array}\right] \\
M_{11}=9 / 4 m_{1} l_{1}+m\left(1 / 4 c_{2} l_{2}+l_{1}^{2}+l_{2} l_{1}\left(c_{1}^{2}-s_{1}^{2}\right)\right) \\
+m_{3}\left(c_{2} l_{2}^{2}+l_{2}^{2}+2 c_{2} l_{1} l_{2}\right) \\
M_{22}=1 / 4 m_{2} l_{2}^{2}+m_{3} l_{2}^{2}+4 / 3 m_{1} l_{1}^{2} \\
M_{23}=M_{32}=m_{3} c_{2} l_{2} \\
M_{33}=m_{3} \\
M_{12}=M_{13}=M_{21}=M_{31}=0 \\
C_{11}=-8 m_{2} l_{1} l_{2} c_{1} s_{1} \dot{q}_{1} \\
+\left(-1 / 2 m_{2} s_{2} C_{2} l_{2}^{2}+m_{3}\left(-2 s_{2} C_{2} l_{2}^{2}-2 s_{2} l_{1} l_{2}\right)\right) \dot{q}_{2} \\
C_{21}
\end{gathered}
$$

where $q \in R^{3}$ and the shorthand notations $c_{1}=\cos \left(q_{1}\right), \quad c_{2}=\cos \left(q_{2}\right), \quad s_{1}=\sin \left(q_{1}\right)$ and 
$s_{2}=\sin \left(q_{2}\right)$ are used.

For the convenience of the simulation, the nominal parameters of the robotic system are given as $m_{1}=3(\mathrm{~kg}) \quad m_{2}=2(\mathrm{~kg}), \quad m_{3}=2.5(\mathrm{~kg}), \quad l_{1}=0.14(\mathrm{~m})$, $l_{2}=0.32(\mathrm{~m})$, and $g=9.8\left(\mathrm{~m} / \mathrm{s}^{2}\right)$ and the initial conditions $\quad q_{1}(0)=1, \quad q_{2}(0)=0, \quad d_{3}(0)=0, \quad \dot{q}_{1}(0)=0$, $\dot{q}_{2}(0)=0, \dot{d}_{3}(0)=0$. The desired reference trajectories are $q_{d 1}(t)=\sin (t), \quad q_{d 2}(t)=\cos (t), \quad d_{d 2}(t)=\cos (t)$, respectively.

The most important parameters that affect the control performance of the robotic system are the external disturbance $t_{l}$, the friction term $f(\dot{q})$, which are injected into the robotic system, and their shapes are expressed as follows:

$$
t_{l}(t)=\left[\begin{array}{lll}
5 \sin (5 t) & 5 \sin (5 t) & 5 \sin (5 t)
\end{array}\right]^{T}
$$

In addition, friction forces are also considered in this simulation and given as

$$
\begin{aligned}
f(\dot{q})= & {\left[20 \dot{q}_{1}+0.8 \operatorname{sgn}\left(\dot{q}_{1}\right) \quad 4 \dot{q}_{2}+2 \operatorname{sgn}\left(\dot{q}_{2}\right)\right.} \\
& \left.4 \dot{d}_{3}+2 \operatorname{sgn}\left(\dot{d}_{3}\right)\right]^{T}
\end{aligned}
$$

In order to exhibit the superior control performance of the proposed SOSICM control system, the control system standalone CMAC is introduced in Figure $\mathbf{3}$ and examined in the mean time [28]. They are applied to control three-link De-icing robot manipulator and the same setting of SOSICM and standalone CMAC control system are chosen as follows: The inputs space of S-CMAC are $d_{s 1}, d_{s 2}$ and $d_{s 3}$, the mean and variance of Gaussian basic functions are selected to cover the input space $\left\{\left[\begin{array}{ll}-1 & 1\end{array}\right]\left[\begin{array}{ll}-1 & 1\end{array}\right]\left[\begin{array}{ll}-1 & 1\end{array}\right]\right\}$; all initial weights are set to zero, i.e., $w_{k 1}=w_{k 2}=w_{k 3}=0, \quad k=1,2, \cdots n_{k i}$. The parameter $\lambda$ in the switching line is one. For recording respective control performance, the mean-square-error of the position-tracking response is defined as:

$$
\text { mse }_{i}=\frac{1}{T} \sum_{j=1}^{T}\left[q_{d i}(j)-q_{i}(j)\right]^{2}, i=1,2,3
$$

where $T$ is the total sampling instant, and $q_{i}$ and $q_{d i}$ are the elements in the vector $q_{i}$ and $q_{d i}$. In this paper, the numerical simulation results carried out by using Matlab software.

Example 1: Consider the standalone CMAC control system is shown in Figure 3.

For the standalone CMAC control system, the parameters are chosen such as: $\beta_{w i}=0.02, \beta_{m i}=0.02$, $\beta_{\sigma i}=0.02, \beta_{n i}=0.02$, the initial value of Gaussian basic functions and scaling factors are defined as $m_{1 i}=-1.0, \quad m_{2 i}=-0.8, \quad m_{3 i}=-0.6, \quad m_{4 i}=-0.4$, $m_{5 i}=-0.2, \quad m_{6 i}=0.0, \quad m_{7 i}=0.2, \quad m_{8 i}=0.4, \quad m_{9 i}=0.6$ $m_{10 i}=0.8, m_{11 i}=1.0, \sigma_{k i}=0.15, k_{1 i}=0.5$ and $k_{2 i}=0.2$ for $k=1,2, \cdots, 11, \quad i=1,2,3$. The simulation results of standalone CMAC system, the responses of joint position and MSE are depicted Figures 7(a)-(f), respectively.

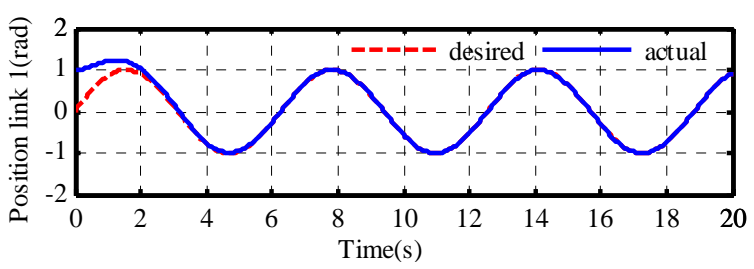

(a)

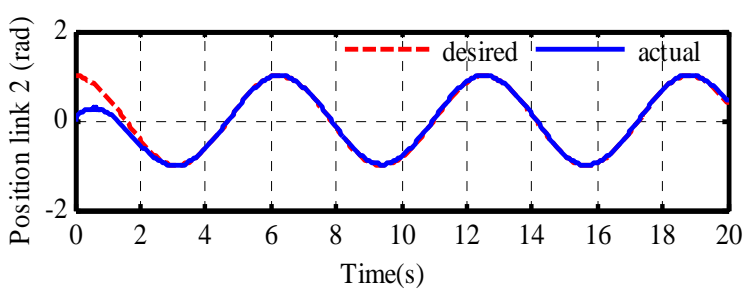

(b)

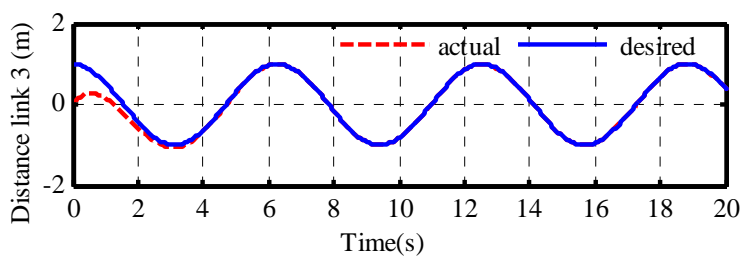

(c)

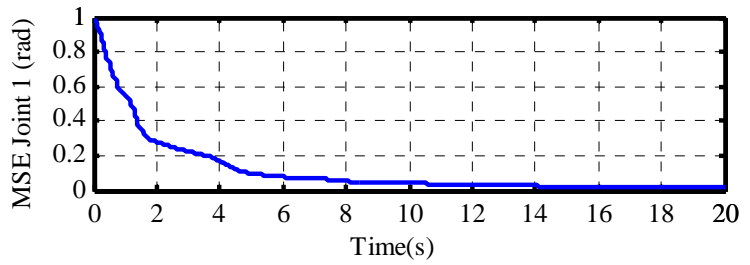

(d)

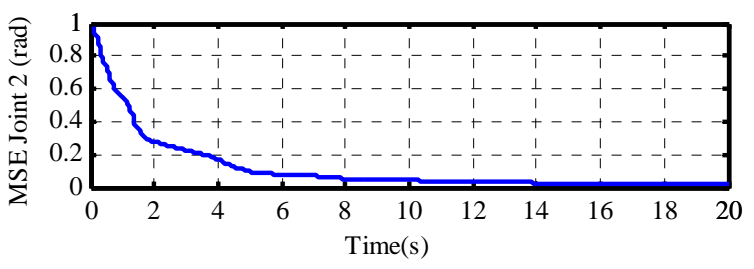

(e)

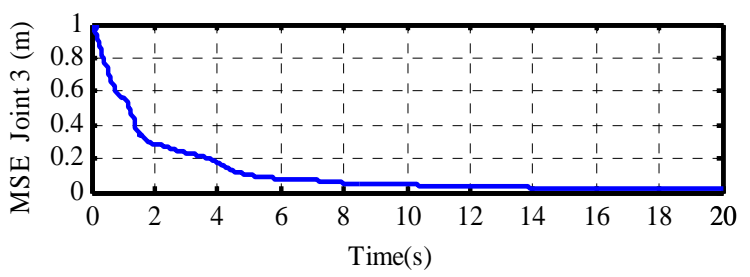

(f)

Figure 7. Simulated position responses and MSE of the Standalone CMAC control system at joints 1, 2 and 3. 
Example 2: Consider the proposed SOSICM control system is shown in Figure 4.

For the proposed SOSICM control system, the parameters are chose in the following:

$\beta_{p i}^{*}=1 /\left[\delta_{p i} / e_{i}(k)\right]^{2}\left\|\partial \tau_{i} / \partial P_{i}\right\|^{2}$ for $P_{i}=w_{k i}, m_{k i}, \sigma_{k i}$ and

$k_{n i}$, and the initial values of system parameters are given as $n_{k i}=2$, the inputs of S-CMAC $d_{s 1}, d_{s 2}$ and $d_{s 3}$ the mean and variance of Gaussian basic functions are selected to cover the input space $\left\{\left[\begin{array}{ll}-1 & 1\end{array}\right]\left[\begin{array}{ll}-1 & 1\end{array}\right]\left[\begin{array}{ll}-1 & 1\end{array}\right]\right\}$. The threshold value of $K_{g i}$ is set as $0.1 ; K_{c i}$ is set as 0.01 for $i=1,2,3$. The simulation results of the proposed SOSICM system, the responses of joint position, MSE and layer number are depicted in Figures 8(a)-(f) and (g), (h) and (k) respectively.

According to the simulation results as shown in Figures $\mathbf{7}$ and $\mathbf{8}$, the joint-position tracking responses of the proposed SOSICM system can be controlled to more closely follow desired reference trajectories than the standalone CMAC as shown in Figure 7 and Figures 8(a)-(c). Our proposed control system for each joint shows that the MSE in Figures 8(d)-(f) is faster than the MSE in Figures 7(d)-(f) and finally converges to 0.009, 0.015 and 0,019 . Meanwhile the MSE of standalone CMAC is $0.032,0.031$ and 0.036 and number of layers of S-CMACs converge to three layers as shown in Figures 8(g), (h) and (k).

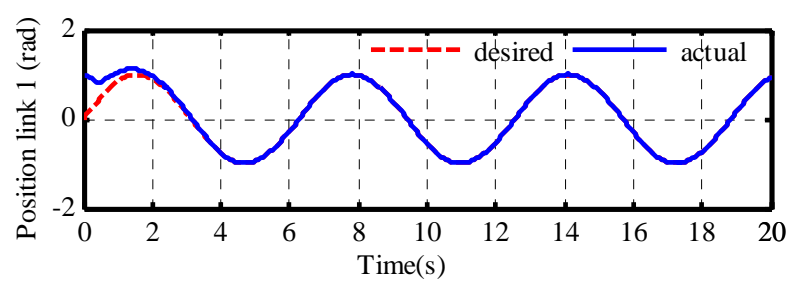

(a)

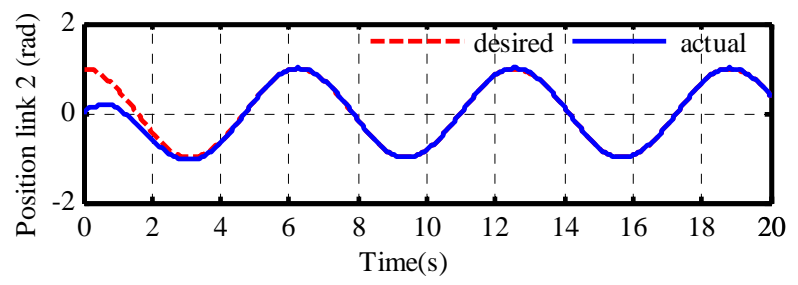

(b)

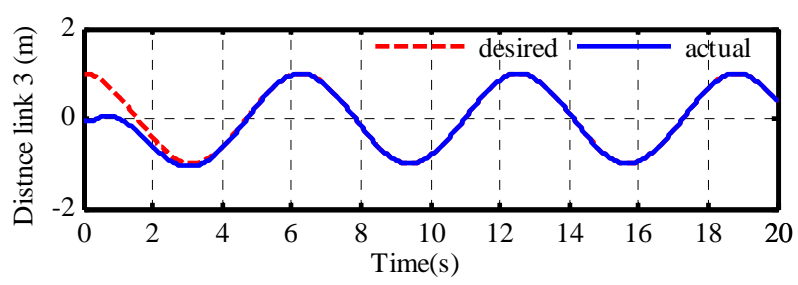

(c)

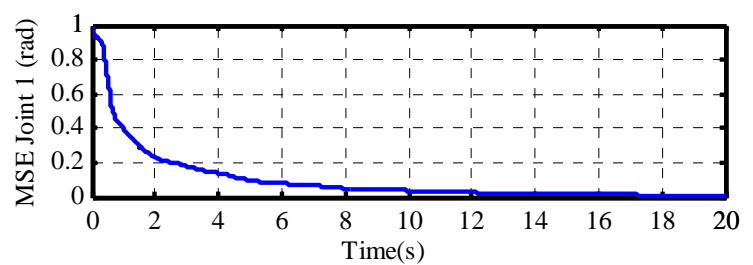

(d)

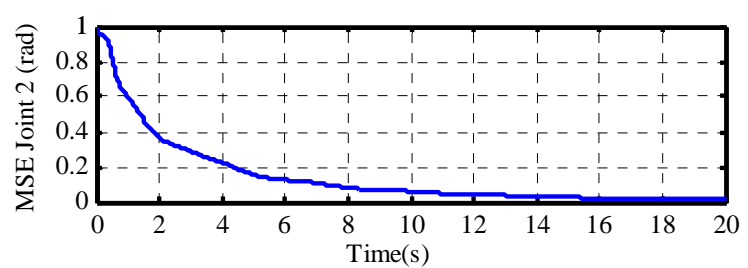

(e)

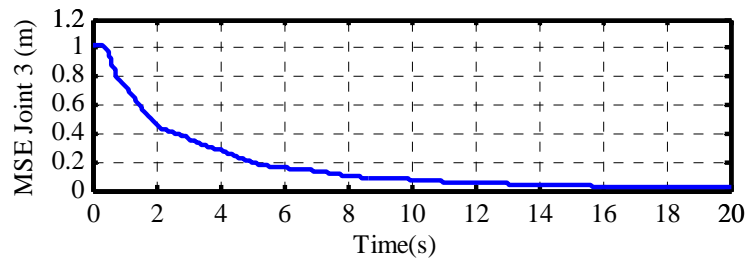

(f)

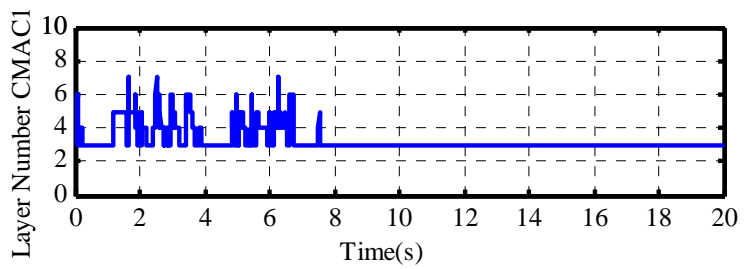

(g)

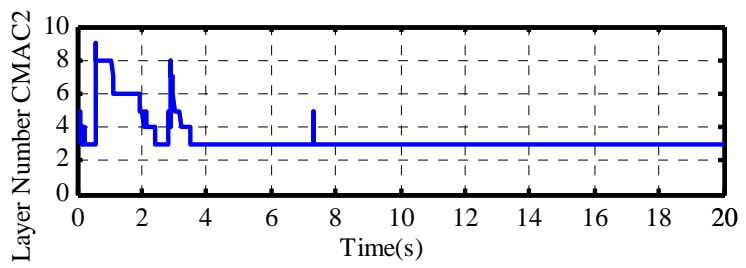

(h)

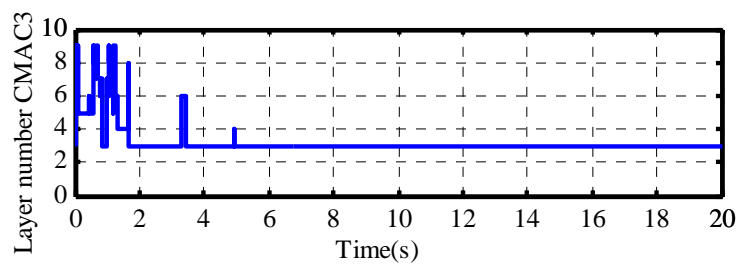

(k)

Figure 8. Simulated position responses, MSEs and layer number of the SOSICM control system at joints 1, 2 and 3.

\section{Conclusions}

In this paper, a SOSICM control system is proposed to 
control the joint position of a three-link De-icing robot manipulator. In the SOSICM system, dynamical system is completely unknown and auxiliary compensated control is not required in the control process. The online tuning laws of S-CMAC parameters are derived in gradient-descent learning method and the discrete-type Lyapunov function is applied to determine the variable optimal learning rates so that the stability of the system can be guaranteed. This paper has successfully developed the SOSICM control system for a three-link De-icing robot manipulator not only requires low memory with online structure and parameters tuning algorithm, but also the input space can be reduced through the signed distance. The simulation results of the proposed SOSICM system can achieve favorable tracking performance for three-link De-icing robot manipulator.

\section{Acknowledgments}

The authors would like to thank the editors and the reviewers for their valuable comments.

\section{References}

[1] A. Vemuri, M. M. Polycarpou and S. A. Diakourtis, "Neural Network Based Fault Detection in Robotic Manipulators,” IEEE Robotics Automation, Vol. 14, No. 2, 1998, pp. 342-348. doi:10.1109/70.681254

[2] W. Z. Gao and R. R. Selmic, "Neural Network Control of a Class of Nonlinear Systems with Actuator Saturation," American Control Conference, Boston, 2006, pp. 147156.

[3] Y. Zou, Y. N. Wang and X. Z. Liu, "Neural Network Robust $\mathrm{H}_{\infty}$ Tracking Control Strategy for robot manipulators,” Applied Mathematical Modelling, Vol. 34, No. 7, 2010, pp. 1823-1838. doi:10.1016/j.apm.2009.09.026

[4] B.-S. Chen, H.-J. Uang and C.-S. Tseng, "Robust Tracking Enhancement of Robot Systems Including Motor Dynamics: A Fuzzy-Based Dynamic Game Approach,” IEEE Transactions on Fuzzy Systems, Vol. 6, No. 4, 1998, pp. 538-552. doi:10.1109/91.728449

[5] H.-X. Li and S.-C. Tong, "A Hybrid Adaptive Fuzzy Control for a Class of Nonlinear MIMO Systems,” IEEE Transactions on Fuzzy Sysemstm, Vol. 11, No. 1, 2003, pp. 24-34. doi:10.1109/TFUZZ.2002.806314

[6] S. Labiod, M. S. Boucherit and T. M. Guerra, "Adaptive Fuzzy Control of a Class of MIMO Nonlinear Systems,” Fuzzy Set and Systems, Vol. 151, No. 1, 2005, pp. 59-77. doi:10.1016/j.fss.2004.10.009

[7] Y. G. Leu, W. Y. Wang, and T. T. Lee, “Observe Based Direct Adaptive Fuzzy Neural Control for Non-Affine Nonlinear Systems," IEEE Transactions on Neural Networks, Vol. 16, No. 4, 2005, pp. 853-861. doi:10.1109/TNN.2005.849824

[8] R. J. Wai and Z. W. Yang, “Adaptive Fuzzy Neural Net- work Control Design via a T-S Fuzzy Model for a Robot Manipulator Including Actuator Dynamics,” IEEE Transactions on Systems, Man and Cybernnetics, Vol. 38, No. 5, 2008, pp. 1326-1346. doi:10.1109/TSMCB.2008.925749

[9] C.-S. Chen, "Dynamic Structure Neural Fuzzy Networks for Robust Adaptive Control of Robot Manipulators," IEEE Transactions on Industrial Electronics, Vol. 55, No. 9, 2008, pp. 3402-3414. doi:10.1109/TIE.2008.926778

[10] B. J. Choi, S. W. Kwak and B. K. Kim, "Design of Single-Input Fuzzy Logic Controller and Its Properties," Fuzzy Sets and Systems, Vol. 106, No. 3, 1999, pp. 299-308. doi:10.1016/S0165-0114(97)00283-2

[11] B. J. Choi, S. W. Kwak and B. K. Kim, "Design and Stability Analysis of Single-Input Fuzzy Logic Controller,” IEEE Transactions on Systems, Man and Cybernnetics, Vol. 30, No. 2, 2000, pp. 303-309. doi:10.1109/3477.836378

[12] K. Ishaque, S. S. Abdullah, S. M. Ayob and Z. Salam, "Single Input Fuzzy Logic Controller for Unmanned Underwater Vehicle,” Journal of Intelligent and Robotic Systems, Vol. 59, No. 3, 2010, pp. 87-100. doi:10.1007/s10846-010-9395-X

[13] J. S. Albus, “A New Approach to Manipulator Control: The Cerebellar Model Articulation Controller," Journal of Dynamic Systems Measurement and Control, Vol. 97, No. 3, 1975, pp. 220-227. doi:10.1115/1.3426922

[14] H. Shiraishi, S. L. Ipri and D. D. Cho, "CMAC Neural Network Controller for Fuel-Injection Systems,” IEEE Transactions on Control Systems Technology, Vol. 3, No. 1, 1995, pp. 32-38. doi:10.1115/1.3426922

[15] S. Jagannathan, S. Commuri and F. L. Lewis, "Feedback Linearization Using CMAC Neural Networks," Automatica, Vol. 34, No. 3, 1998, pp. 547-557. doi:10.1016/S0005-1098(97)00206-9

[16] C. T. Chiang and C. S. Lin, "CMAC with General Basis Functions,” Journal of Neural Networks, Vol. 9, No. 7, 1996, pp. 1199-1211. doi:10.1016/S0005-1098(97)00206-9

[17] Y. H. Kim and F. L. Lewis, "Optimal Design of CMAC Neural-Network Controller for Robot Manipulators,” IEEE Transactions on Systems, Man and Cybernnetics, Vol. 30, No. 1, 2000, pp. 22-31. doi:10.1109/5326.827451

[18] C. M. Lin and Y. F. Peng, "Adaptive CMAC-Based Supervisory Control for Uncertain Nonlinear Systems,” IEEE Transactions on Systems, Man and Cybernnetics, Vol. 34, No. 2, 2004, pp. 1248-1260. doi:10.1109/TSMCB.2003.822281

[19] S. F. Su, T. Tao and T. H. Hung, "Credit Assigned CMAC and Its Application to Online Learning Robust Controllers," IEEE Transactions on Systems, Man and Cybernnetics, Vol. 33, No. 2, 2003, pp. 202-213. doi:10.1109/TSMCB.2003.810447

[20] H.-C. Lu, C.-Y. Chuang and M.-F. Yeh, "Design of Hybrid Adaptive CMAC with Supervisory Controller for a Class of Nonlinear System,” Neurocomputing, Vol. 72, No. 7-9, 2009, pp. 1920-1933. 
doi:10.1016/j.neucom.2008.07.004

[21] Y. F. Peng and C. M. Lin, "Intelligent Hybrid Control for Uncertain Nonlinear Systems Using a Recurrent Cerebellar Model Articulation Controller," IEEE Proceedings Control Theory and Applications, Vol. 151, No. 5, 2004. pp. 589-600. doi:10.1049/ip-cta:20040903

[22] J. Hu and F. Pratt, "Self-Organizing CMAC Neural Networks and Adaptive Dynamic Control,” IEEE International Symposium on Intelligent Control/Intelligent Systems and Semiotics, Cambridge, 1999, pp. 259-265.

[23] H. C. Lu and C. Y. Chuang, "Robust Parametric CMAC with Self-Generating Design for Uncertain Nonlinear Systems,” Neurocomputing, Vol. 74, No. 4, 2011, pp. 549-562. doi:10.1016/j.neucom.2010.09.001

[24] H. M. Lee, C. M. Chen and Y. F. Lu, “A Self-Organizing HCMAC Neural-Network Classifier,” IEEE Transactions on Neural Networks, Vol. 14, No. 1, 2003, pp. 15-27.
doi:10.1109/TNN.2002.806607

[25] C. M. Lin and T. Y. Chen, "Self-Organizing CMAC Control for a Class of MIMO Uncertain Nonlinear Systems," IEEE Transactions on Neural Networks, Vol. 20, No. 9, 2009, pp. 1377-1384. doi:10.1109/TNN.2009.2013852

[26] M.-F. Yeh, “Single-Input CMAC Control System,” Neurocomputing, Vol. 70, No. 16-18, 2007, pp. 2638-2644. doi:10.1016/j.neucom.2006.05.019

[27] M.-F. Yeh, H.-C. Lu and J.-C. Chang, "Single-Input CMAC Control System with Direct Control Ability,” IEEE Transactions on Systems, Man and Cybernnetics, Vol. 3, No. 1, 2006, pp. 2602-2607. doi:10.1109/ICSMC.2006.385256

[28] M.-F. Yeh and C.-H. Tsai, "Standalone CMAC Control Systems with Online Learning Ability,” IEEE Transactions on Systems, Man and Cybernnetics, Vol. 40, No. 1, 2010, pp. 43-53. doi:10.1109/TSMCB.2009.2030334 